\title{
Risiko des Eintrags von Phosphor in den Hallwilersee durch Bodenerosion
}

\author{
S. Müller ${ }^{1, a}$ and D. Schaub ${ }^{1}$ \\ ${ }^{1}$ Departement Bau, Verkehr und Umwelt, Entfelderstr. 22, 5001 Basel, Switzerland

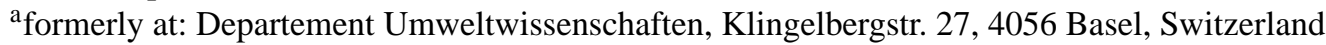 \\ Correspondence to: S. Müller (sonja.mueller@ag.ch)
}

Received: 28 May 2014 - Accepted: 24 July 2014 - Published: 13 August 2015

\begin{abstract}
Kurzfassung. An important factor in the release of phosphorus by soil erosion, with corresponding consequences on the quality of surface waters, is the formation of aggregates and their stability. Around the eutrophic Lake Hallwil six arable lands were examined in five repetitions regarding aggregate and particle size distribution, P-contents of the different fractions and aggregate stability. Central to this was the use of the setting column for fractionation of soil samples. In the case of Lake Hallwil the risk of phosphorus discharges by soil erosion seems low since the phosphorus is mainly bound in aggregates which are transported over short distances only. Thus other pathways (runoff from grassland, leaching via drains) may be more important.
\end{abstract}

\section{Einleitung}

Die übermässige Belastung der Schweizer Mittellandseen mit Phosphor (P) stellt seit Jahren ein grosses Problem dar, da es sich um einen limitierenden Nährstoff handelt. Folgen eines Überschusses dieses Nährstoffs können Algenblüten, Sauerstoffmangel und infolge dessen Fischsterben sein. Für die Belastung verantwortlich gemacht wird insbesondere die Landwirtschaft wegen Drainagen, Bodenerosion und der Nährstoffabschwemmung von Grasland (Prasuhn, 2010).

Im Hallwilersee liegt eine übermässige P-Belastung während über 100 Jahren vor. See-externe Massnahmen wie die Abwasserreinigung und der finanzielle Anreiz für Landwirte zur Reduktion der P-Düngung im Rahmen des Phosphorprojekts der Kantone Aargau und Luzern stellen wichtige Werkzeuge zur Gesundung des Hallwilersees dar. Die jährliche PBelastung des Sees konnte von rund 16 tP Mitte der 1970er Jahre auf $3.5 \mathrm{tP}$ im Jahr 2008 reduziert werden (Stöckli, 2010). Als see-interne Massnahme wird der See seit 1986 künstlich belüftet. Im Winter wird Druckluft als Zirkulationshilfe und im Sommer Sauerstoff zur Belüftung ins Tiefenwasser gepumpt.

Innerhalb des Einzugsgebiets des Hallwilersees wurden sechs Ackerböden auf ihre Phosphorkonzentration untersucht. Das Ziel bestand darin, den Boden mittels Setzsäule in unterschiedliche Aggregatklassen zu fraktionieren und da- mit das Risiko für den Austrag von $\mathrm{C}$ und $\mathrm{P}$ in Vorfluter und Seen abzuschätzen. Es wird angenommen, dass der P-Eintrag in den Hallwilersee nur zu einem kleinen Teil aus der Erosion landwirtschaftlich genutzter Flächen stammt. Der Hauptteil wird in der Abschwemmung von Gülle und Dünger von Landwirtschaftsflächen vermutet.

\section{Untersuchungsgebiet}

Das Untersuchungsgebiet befindet sich im Schweizer Mittelland rund um den Hallwilersee. Das Mittelland ist sowohl die bevölkerungsstärkste Region der Schweiz, als auch der wirtschaftliche Schwerpunkt des Landes. Es bildet den wichtigsten Siedlungs- und Landwirtschaftsraum der Schweiz. Aus diesem Grund sind rund um den Hallwilersee, welcher eine Fläche von $10.29 \mathrm{~km}^{2}$ und ein Einzugsgebiet von rund $170 \mathrm{~km}^{2}$ aufweist, zahlreiche Landwirtschaftsflächen zu finden. Die östliche Seeseite, die etwas weniger steil ist, weist eine intensivere Landwirtschaft auf als die westliche Seite.

Geologisch betrachtet liegt das Mittelland im Bereich des Molassemeeres, wodurch hauptsächlich Gesteine wie Nagelfluh, Mergel und Sandsteine zu finden sind. Überprägt wurde das ganze Gebiet durch die Vergletscherung. Während des Pleistozäns waren grosse Teile des Mittellands von Gletschern bedeckt, welche sich mehrfach ausdehnten und wie- 


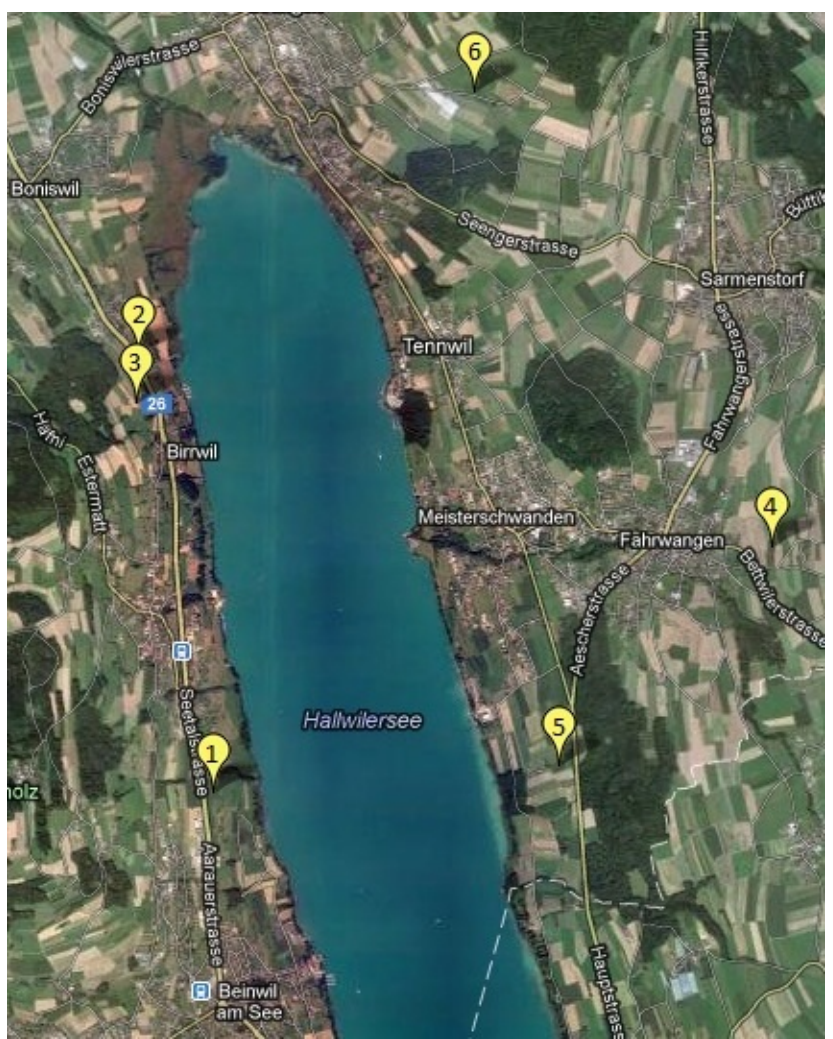

Abb. 1. Probestandorte.

der zurückzogen. Das Gebiet des Hallwilersees war während der Würm-Eiszeit mit einer $600-700 \mathrm{~m}$ dicken Eisschicht bedeckt. Noch heute sind zahlreiche Moränen aus unterschiedlichen Phasen der würmzeitlichen Vergletscherung durch den Reussgletscher sichtbar (Annaheim et al., 1958). Die drei grossen Mittellandseen Baldeggersee, Sempachersee und Hallwilersee sind alle nach der letzten Eiszeit aus Toteislöchern entstanden.

Die Böden entwickelten sich in erster Linie aus Moränenmaterial aus der Würmeiszeit. Es handelt sich vorwiegend um Braunerden, Parabraunerden und Gleye, welche grossflächig eine relativ homogene Verteilung aufweisen. Oft handelt es sich um Lehme, welche relativ schlecht durchlässig sind und mit Drainagen entwässert werden (Prasuhn, 2010). Die Mächtigkeit der Böden variiert zwischen mässig tiefgründig bis tiefgründig.

\section{Methoden}

Für die Untersuchungen wurden sechs Standorte ausgewählt (Abb. 1), deren Auswahl auf verschiedenen Faktoren basierte. Sie sollten alle in Hanglage sein, dieselbe Vorkultur (in diesem Fall Mais) haben und in den letzten Monaten nicht gedüngt worden sein. Pro Standort wurden fünf Messpunkte (Wiederholungen) definiert, um welche in einem Radius von einem Meter mit einer kleinen Schaufel fünfmal ca. $300 \mathrm{~g}$
Oberboden bis zu einer Tiefe von max. $8 \mathrm{~cm}$ entnommen wurden. Aufgrund des starken Regens in den Vortagen war der Boden sehr feucht bis nass.

Die Proben wurden im Labor mehrere Tage im Trockenschrank bei $40{ }^{\circ} \mathrm{C}$ getrocknet und anschliessend auf kleiner $8 \mathrm{~mm}$ gesiebt. Die jeweils fünf Proben wurden danach zu einer Mischprobe pro Messpunkt vermengt (also fünf Wiederholungen pro Standort, d.h. insgesamt 30 Proben).

Die Proben wurden mittels Setzsäule, deren Prinzip auf dem Stokes'schen Gesetz basiert, in fünf Klassen fraktioniert. Die verwendeten Setzzeiten ergeben sich aus den Standard-Siebgrössen für die Nasssiebung: 250, 125, 63 und $32 \mu \mathrm{m}$. Die entsprechenden Setzzeiten sind $40 \mathrm{~s}, 2 \mathrm{~min}$, $10 \mathrm{~min}$ und $30 \mathrm{~min}$ und wurden aufgrund der Stokes'schen Gleichung errechnet. Material, welches sich nach 30min noch nicht gesetzt hat, ist kleiner als $32 \mu \mathrm{m}$ und wird als eine separate Klasse behandelt (Hübner, 2011; Dearnaley, 1996).

Die einzelnen Fraktionen wurden getrocknet, ausgewogen und anschliessend für die weiteren Untersuchungen auf Pund C-Gehalt, Korngrössen und Aggregatstabilität verwendet. Die Korngrössenanalyse wurde im aggregierten und im dispergierten Zustand mittels Mastersizer 2000 von Malvern durchgeführt.

Der totale Phosphorgehalt wurde nach der Methode von Saunders und Williams (1955), verändert nach Gallet et al. (2003) bestimmt. Es wurden $0.5 \mathrm{~g}$ Boden im Muffelofen bei $540^{\circ} \mathrm{C}$ während einer Stunde verglüht. Die Rückstände wurden im Exsikkator ausgekühlt und zurückgewogen. Anschliessend wurde der Boden mit $25 \mathrm{~mL}$ 0.5M Schwefelsäure $\mathrm{H}_{2} \mathrm{SO}_{4}$ vermischt und über Nacht auf dem Schüttler extrahiert. Danach wurden die Proben und $0.5 \mathrm{~mL}$ der Lösung mit $9.5 \mathrm{~mL} \mathrm{H}_{2} \mathrm{O}^{-}$verdünnt und anschliessend photometrisch mit Molybdänblau bestimmt.

Für die Messung der Aggregatstabilität gibt es aufgrund der unterschiedlichen Zerfallsmechanismen von Aggregaten diverse Messmethoden. Für diese Arbeit wurde die von Kemper und Rosenau (1986) beschriebene „Nasse-SiebMethode“" von Eijkelkamp Agrisearch Equipment gewählt. Das Gerät besteht aus acht Sieben mit einer Maschengrösse von $250 \mu \mathrm{m}$, acht Behältern und einem Schüttelapparat (Eijkelkamp Agrisearch Equipment, 2008).

Es wurden $4 \mathrm{~g}$ Boden mit einem Durchmesser von 1-2 mm in die Siebe gegeben und mittels Sprühflasche angefeuchtet. Anschliessend wurden die Siebe mit den Aggregaten vollständig in die Behälter eingetaucht. Während des Siebvorgangs wurden die Siebe 20 min lang mit einer Frequenz von 34 Mal pro Minute auf- und abwärts bewegt. Nach dem Siebvorgang befanden sich jene Aggregate, welche eine geringe Stabilität aufweisen und deshalb zerfallen sind, in den Behältern. In den Sieben befanden sich die stabilen Aggregate. Das Wasser mit den instabilen Aggregaten sowie die stabilen Aggregate auf dem Sieb wurden je in einen separaten Behälter gegeben und nach dem Trocknen bei $40^{\circ} \mathrm{C}$ im Trockenschrank ausgewogen. Die Aggregatstabilität errechnet sich aus dem Gewicht der stabilen Aggregate geteilt durch die 


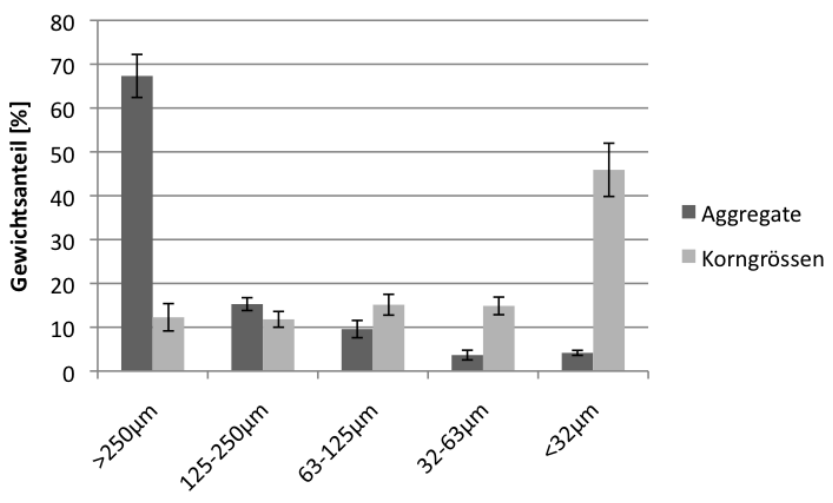

Abb. 2. Prozentualer Gewichtsanteil pro Aggregat- und Korngrössenfraktion mit Standardabweichung.

Summe der stabilen und der instabilen Aggregate (Eijkelkamp Agrisearch Equipment, 2008).

\section{Resultate und Diskussion}

\subsection{Aggregat- und Korngrössenverteilung}

Sowohl bei der Fraktionierung der Aggregate mittels Setzsäule als auch in der Korngrössenverteilung wurden lediglich geringfügige Unterschiede zwischen den einzelnen Flächen gefunden und es ergab sich ein sehr homogenes Bild über alle sechs Untersuchungsflächen.

Abbildung 2 zeigt den prozentualen Gewichtsanteil pro Fraktion. Als Aggregatfraktion gilt der gemessene Wert im aggregierten Zustand, die Korngrössenfraktion stellt den Messwert im dispergierten Zustand dar. Es wurde jeweils der durchschnittliche Gewichtsanteil der einzelnen Fraktionsklassen sowohl für die Aggregate als auch für die Korngrössen über alle Messpunkte und Flächen berechnet. Es ist deutlich zu sehen, dass die Verteilung der Aggregate und jene der Korngrössen gegenläufig sind. Die Verteilung der Aggregate aufgrund der Setzsäulen-Fraktionierung zeigt eine starke Gewichtszunahme mit grösser werdenden Aggregaten. Zwischen 60 und $72 \%$ des Materials setzen sich jeweils während den ersten $40 \mathrm{~s}$ ab. Rund 14-17\% haben sich während des zweiten Intervalls von 40 bis $120 \mathrm{~s}$, und 8-13\% des Materials während des dritten Intervalls von 120 bis 600 s gesetzt. Entsprechend gering ist der Gewichtsanteil der feinsten Fraktionen, welche sich zwischen 10 und 30 min respektive nach 30 min gesetzt haben. Diese Fraktionen machen einen Gewichtsanteil von jeweils 3-5\% aus.

Die Gewichtsverteilung der mineralischen Partikel, also der Korngrössen, zeigt einen gegensätzlichen Verlauf. Während sich die Gewichtsanteile der Korngrössen-Fraktionen $>32 \mu \mathrm{m}$ zwischen rund $12-15 \%$ bewegen, macht das Gewicht der Feinschluff- und Tonpartikel mit einem Durchmesser $<32 \mu \mathrm{m} 46 \%$ aus.

Abbildung 3 zeigt die Korngrössenverteilung innerhalb der einzelnen Aggregatfraktionen, welche mittels Nasssie-

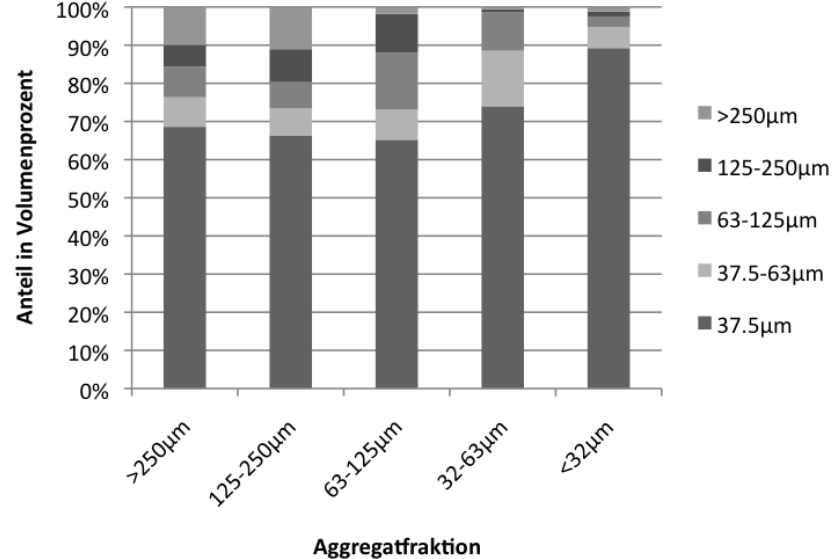

Abb. 3. Anteil der Korngrössen an den Aggregaten. Grössere Korngrössen als Aggregate ergeben sich aus der Bestimmung von Korngrösse und Aggregatsgrösse mit unterschiedlichen Methoden.

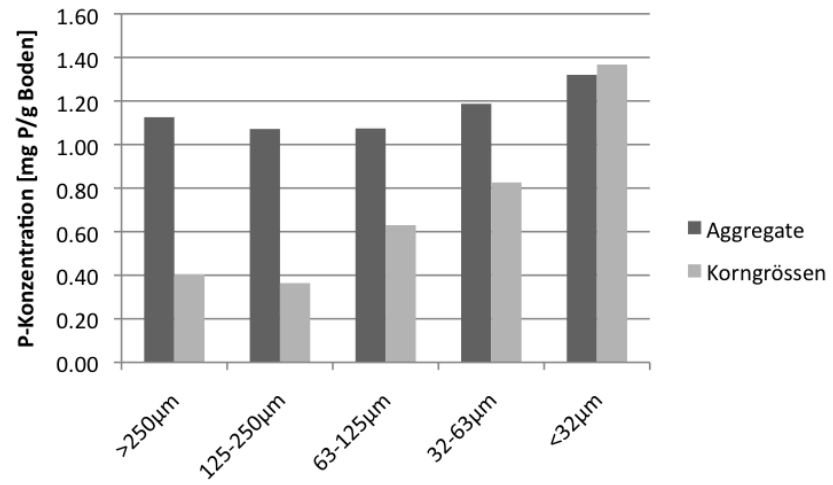

Abb. 4. P-Konzentration pro Aggregat- und Korngrössenfraktion.

bung ermittelt wurde. Alle Fraktionen weisen einen sehr hohen Anteil an Partikeln $<32 \mu \mathrm{m}$ auf. Sie machen jeweils zwischen 68 und $89 \%$ aus. Diese geringen Unterschiede sind wichtig zur Interpretation der P-Konzentration der einzelnen Fraktionen (vgl. Kap. 4.2).

\subsection{Phosphor}

Abbildung 4 zeigt die Verteilung der P-Konzentration in den einzelnen Fraktionen der Aggregate sowie der mittels Nasssiebung erhobenen Korngrössen. Die mittlere GesamtPhosphor-Konzentration auf den sechs Untersuchungsflächen bewegt sich zwischen $820 \mathrm{mg} \mathrm{P} \mathrm{kg}^{-1}$ TS (Fläche 5) und $1380 \mathrm{mg} \mathrm{P} \mathrm{kg}^{-1}$ TS (Fläche 1). Dies belegt die gebietstypische Spanne der P-Gehalte der Böden im Untersuchungsgebiet aufgrund der Lage der Standorte und ihrer bisherigen Nutzung. Die höchsten P-Konzentrationen wurden sowohl in den Aggregat-, als auch in den Korngrössenfraktionen $<32 \mu \mathrm{m}$ gefunden. Allgemein lässt sich sagen, je kleiner die Aggregate resp. Korngrösse, desto höher die PKonzentration. Allerdings ist diese Zunahme weitaus weni- 


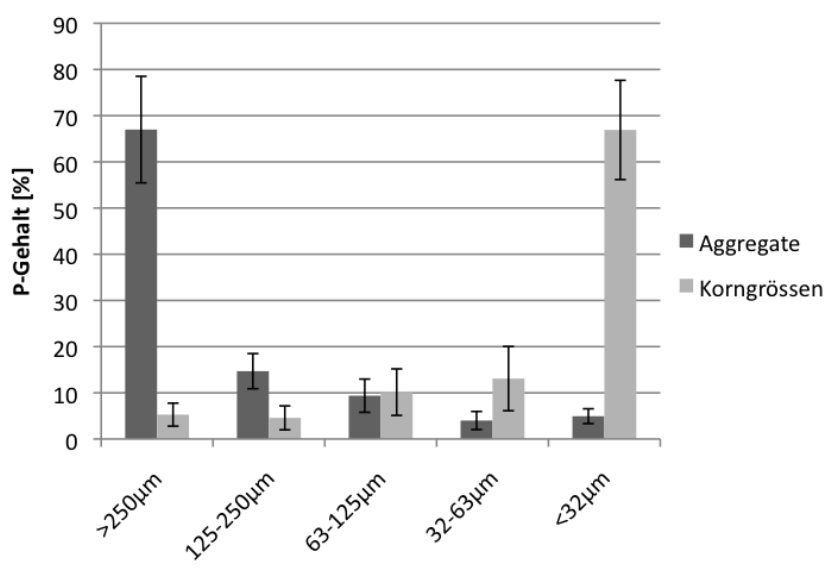

Abb. 5. Prozentualer P-Gehalt pro Aggregat- und Korngrössenfraktion mit Standardabweichung.

ger gross für die Aggregatgrösse, als für die Korngrössen mineralischer Partikel, welche einen enormen Anstieg der Konzentrationen mit abnehmender Korngrösse zeigt.

Wird der in Abb. 5 dargestellte prozentuale Gehalt an Phosphor betrachtet, ist wie bereits bei der Gewichtsverteilung eine gegenläufige Verteilung der Aggregat- und der Korngrössenfraktionen zu erkennen. Während der Gehalt bei den Korngrössenfraktionen sowohl vom Gewicht als auch von der Konzentration beeinflusst wird und eine starke $\mathrm{Zu}$ nahme des Gehalts mit abnehmender Korngrösse zu beobachten ist, wird der P-Gehalt bei den Aggregaten aufgrund der geringen Konzentrationsunterschiede zwischen den einzelnen Fraktionen lediglich vom Gewicht bestimmt. Folglich binden die grossen Aggregate mehr P als die kleinen. Aufgrund des hohen Gewichtanteils der Aggregate $>250 \mu \mathrm{m}$ fällt besonders diese Fraktion stark ins Gewicht.

Prasuhn und Braun (1994) haben für den Kanton Bern die Phosphorverluste aus diffusen Quellen in die Oberflächengewässer abgeschätzt. Sie haben aus Literaturwerten von pflanzenverfügbaren P-Gehalten in Böden Gesamt-Phosphor-Konzentrationen $\left(\mathrm{P}_{\text {tot }}\right)$ zwischen 500 und $1100 \mathrm{mg} \mathrm{P} \mathrm{kg}^{-1} \mathrm{TS}$ berechnet. Die hier gefundenen Gehalte (820-1380 mg P kg${ }^{-1} \mathrm{TS}$ ) liegen höher. Dies lässt zunächst einmal auf höhere P-Einträge in Oberflächengewässer im Falle von Bodenerosionsereignissen schliessen. Schwieriger abzuschätzen ist jedoch die ökologische Wirkung, da aus höheren $\mathrm{P}_{\text {tot }}$-Gehalten nur bedingt auf hohe pflanzenverfügbare P-Gehalte geschlossen werden kann (BUWAL, 2004). Daher kann aus den $\mathrm{P}_{\text {tot }}$-Gehalten der untersuchten Böden auch kein Rückschluss auf eine allfällige Überdüngung gezogen werden, woraus sich Konsequenzen für die künftige Düngungspraxis ergeben würde. Zur gleichen Einschätzung kommt Baltzer (2012) aufgrund einer Auswertung von 1'500 Bodenproben aus Landwirtschaftsflächen im Einzugsgebiet des Hallwilersees.

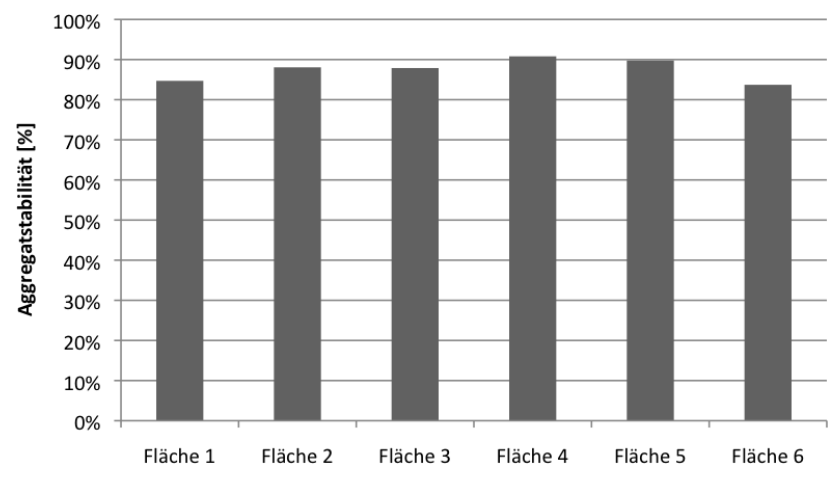

Abb. 6. Aggregatstabilität pro Fläche.

\subsection{Aggregatstabilität}

Die mittlere Aggregatstabilität weist eine geringe Variabilität zwischen den einzelnen Flächen auf (vgl. Abb. 6). Auf Fläche 4 ist die Aggregatstabilität mit $90.8 \%$ am höchsten, Fläche 6 weist mit $83.7 \%$ die geringste Stabilität der Aggregate auf. Der Durchschnitt über sämtliche Messpunkte $(N=30)$ beträgt $87.5 \%$. Wichtig ist zu berücksichtigen, dass die Proben im November genommen wurden. Aggregatstabilität ist eine variable Grösse und verändert sich mit der Jahreszeit und der Bearbeitung (Amézketa, 1999).

Die Aggregatstabilität gibt Hinweise auf das Verhalten eines Bodens unter den Einflüssen von Wasser, Wind und der Bewirtschaftung. Sie drückt aus, wie stark der Zusammenhalt der Aggregate bei einer von aussen einwirkenden Kraft ist (Amézketa, 1999). Die Aggregatstabilität ist auch ein wichtiger Faktor für die Aggregatverteilung. Würden die Böden eine geringe Aggregatstabilität aufweisen, wäre es in der Setzsäule zu einem stärkeren Zerfall gekommen. Dies wiederum hätte den Anteil der kleineren Aggregate erhöht. Durch die hohe Aggregatstabilität kam es jedoch nur zu einem geringen Aggregatzerfall. Auf die Untersuchungsflächen übertragen bedeutet dies im Falle eines Niederschlagsereignisses einen geringen Aggregatzerfall und somit ein geringes Erosionsrisiko. Dies steht im Widerspruch zur Erosionsrisikokarte des Bundesamts für Landwirtschaft (BLW, 2010), in welcher das Hallwilersee-Einzugsgebiet eine hohe potentielle Erosionsgefährdung aufweist. Bei den Feldbegehungen konnten keine sichtbaren Spuren von Bodenerosion festgestellt werden, obwohl es in den Tagen und Wochen vor der Untersuchung zu starken Niederschlägen gekommen war. Auch die Landwirte der untersuchten Standorte gaben an, aktuell oder in der Vergangenheit noch nie Probleme mit Erosion gehabt zu haben. Somit kann davon ausgegangen werden, dass die Aggregatstabilität ein sehr wichtiger Faktor bezüglich Erosion ist und insbesondere in Gebieten mit Hanglage ein sehr wichtiger Faktor ist. 


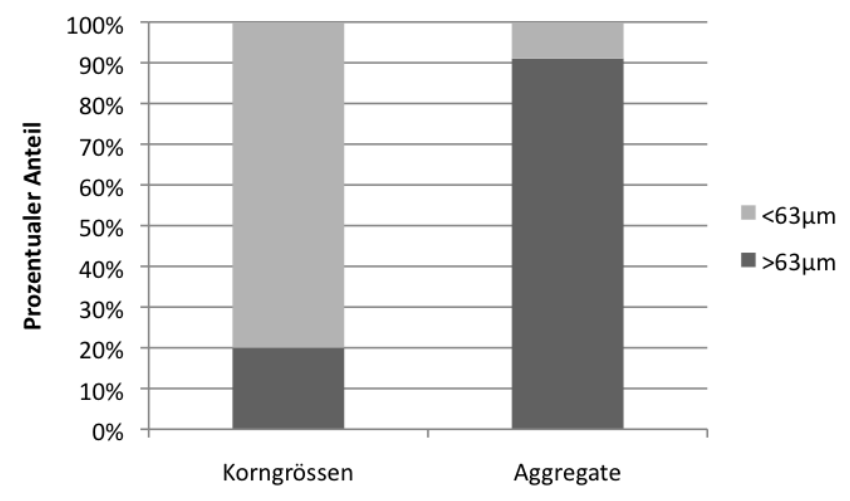

Abb. 7. Prozentualer Anteil von P an erosionsanfälligen Bodenpartikeln und Aggregaten.

\subsection{Diskussion}

Anfällig auf Erosion sind laut Starr et al. (2000) jene Partikel und Aggregate kleiner als $63 \mu \mathrm{m}$. Betrachtet man den prozentualen Anteil an Phosphor, welcher in den Bodenteilchen dieser Grösse gebunden ist (Abb. 7), sind grosse Unterschiede zwischen den Aggregaten und den Korngrössen zu sehen. Knapp $80 \%$ des Phosphors sind in mineralischen Partikeln $<63 \mu \mathrm{m}$ gebunden und sind so erosionsgefährdet. Durch die Aggregation werden diese Partikel jedoch in Aggregate unterschiedlicher Grösse eingebunden. Der Anteil an P, welcher in Aggregaten $<63 \mu \mathrm{m}$ gebunden und somit von Erosion betroffen ist, beträgt demnach noch lediglich knappe $10 \%$. Es befinden sich also $90 \%$ des Gesamt-Phosphors in Aggregaten grösser als $63 \mu \mathrm{m}$. Diese werden zwar von Bodenerosion erfasst, aber aufgrund des höheren Gewichts und der höheren Dichte nach kurzer Transportstrecke wieder abgelagert und so in der Landschaft umverteilt.

Durch die Aggregation der einzelnen Bodenteilchen zu Aggregaten kommt es zu einer Verteilung der feinen Bodenpartikel und somit des adsorbierten Phosphors in sämtlichen Aggregatfraktionen. Aus diesem Grund sind die Konzentrationsunterschiede von $\mathrm{P}$ in den einzelnen Aggregatfraktionen nicht so stark ausgeprägt wie bei den Korngrössenfraktionen, wie die Resultate gezeigt haben. Deshalb ist entgegen der Aussagen von Auerswald (1989) sowie Sharpley et al. (1981) bei den hier untersuchten Böden nicht mit einer Anreicherung von Phosphor im Erosionsmaterial zu rechnen. Wichtig hierbei ist auch die Erkenntnis von Starr et al. (2000), dass mindestens $73 \%$ des mobilisierten Materials wieder in der Landschaft deponiert werden und nicht bis in die Gewässer transportiert werden. Bei Prasuhn und Braun (1994) ist von $80 \%$ die Rede. Im Fall der vorliegenden Arbeit sind rund $90 \%$ der gesamten Bodenprobe Aggregate $>63 \mu \mathrm{m}$ und werden somit wieder abgelagert, bevor sie in die Gewässer gelangen, solange keine starke lineare Erosion auftritt. Rillenerosion kann auch grössere Aggregate über weite Distanzen verfrachten. Auch $90 \%$ des Gesamtphosphors sind in jenen Aggregaten gespeichert, welche vor Erreichen eines
Gewässers abgelagert werden. Zu beachten gilt jedoch, dass die Untersuchung auf einer Probe des gesamten Oberbodens basiert. Es handelt sich also nicht um Material, welches aufgrund eines Niederschlagsereignisses tatsächlich mobilisiert wurde und kann somit nicht vorbehaltlos mit den Untersuchungen von Starr et al. (2000) verglichen werden.

Beim Hallwilersee stellt sich nun die Frage, welche anderen Ursachen neben der Erosion für den Phosphoreintrag verantwortlich sind und wie dieser Eintrag verhindert werden kann. Prasuhn und Braun (1994) nehmen an, dass diffuse Quellen rund $70 \%$ des Phosphorverlustes ausmachen, gegenüber $30 \%$ aus punktuellen Quellen. Von den diffusen Quellen kommt neben Erosion auch Abschwemmung von Grünland, Bachbetterosion, zu P aus dem Laubfall und Auswaschung unter Wald in Frage. Wenn also die Erosion nicht den Haupteintrag bildet, muss entweder die Abschwemmung, die Auswaschung oder der Drainagenabfluss dafür verantwortlich sein. Drainagen leiten Niederschlagswasser bereits innerhalb der obersten $100 \mathrm{~cm}$ des Bodens in ein Oberflächengewässer. Die mit dem Drainageabfluss erfassten Nährstoffe haben somit nur eine kurze Strecke, um sich an Bodenpartikel im Gerinne zu binden. Daher ist Drainagenwasser meist nährstoffreich. Im Einzugsgebiet des Hallwilersees sind zahlreiche Flächen drainiert Aus diesem Grund dürften die Drainagen ein wichtige Rolle bei der PVerlagerung spielen.

Ein wichtiger Prozess beim P-Eintrag in die Gewässer ist die Adsorption von gelöstem Phosphor an Bodenteilchen. Dieser Prozess spielt insbesondere bei Niederschlagsereignissen eine bedeutende Rolle, weil dadurch der Anteil des gelösten P reduziert wird. Das transportierte Bodenmaterial stellt somit eine P-Senke und nicht eine P-Quelle dar. Entscheidend ist bei diesem Prozess die selektive Erosion feiner Partikel, die eine höhere Adsorptionskapazität für P aufweisen (Barbosa et al., 2009). Mit zunehmender Konzentration an suspendiertem Sediment im Abfluss nimmt daher die Konzentration des gelösten Phosphors ab. Entscheidend ist dabei die Aggregation des Bodenmaterials. Je stärker ein Boden aggregiert ist, desto geringer ist der Anteil der nicht-aggregierten suspendierten Tonpartikel und somit auch die Adsorption des gelösten Phosphors. Für die untersuchten Flächen bedeutet dies, dass die Fähigkeit, bei einem Niederschlagsereignis gelösten Phosphor an Bodenteilchen zu binden, relativ gering ist, da die Böden eine hohe Aggregatstabilität aufweisen und somit wenig nicht-aggregierte Ton- und Feinschluffpartikel vorhanden sind.

Da der erosive Eintrag von Phosphor in den Hallwilersee aufgrund der starken Aggregation und der Konzentration des Phosphors in den grösseren Aggregaten eher gering ist, muss die Abschwemmung eine wichtigere Rolle spielen. Um diese Annahme zu beweisen, wären weitere Untersuchungen in diese Richtung von Interesse. 
5 Fazit

Ein wichtiger Faktor bei der Freisetzung von Phosphor durch Bodenerosion ist die Bildung von Aggregaten sowie deren Stabilität. Feine phosphorreiche Bodenpartikel werden durch Aggregation in Mikro- oder Makroaggregaten eingebunden. Aufgrund ihres höheren Gewichts und der selektiven Erosion von Material $<63 \mu \mathrm{m}$ sind Aggregate weniger erosionsanfällig als Einzelpartikel.

Die hohe Aggregation und Aggregatstabilität der Böden deuten darauf hin, dass der Eintrag von Phosphor in den Hallwilersee über flächenhafte Erosion gering ist. Lineare Erosion kann allerdings grössere Aggregate transportieren, sodass die hohen P-Gehalte der Böden auf diese Weise zu hohen PEinträgen führen.

Folglich müssen noch andere Quellen für den Eintrag verantwortlich sein. Eine mögliche Ursache ist beispielsweise die Abschwemmung aus Grasland. Insbesondere bei Starkniederschlägen direkt nach dem Ausbringen von Gülle oder Mist ist die Gefahr der Abschwemmung durch Oberflächenabfluss oder die Auswaschung über Drainagen hoch und die entsprechende Phosphor-Fracht dürfte enorm sein.

Edited by: P. Greenwood

Reviewed by: two anonymous referees

\section{Literatur}

Amézketa, E.: Soil Aggregate Stability: A review, J. Sustain. Agr., 14, 83-151, 1999.

Annaheim, H., Bögli, A., and Moser, S.: Die Phasengliederung der Eisrandlagen des würmeiszeitlichen Reussgletschers im zentralen Schweizerischen Mittelland, Geogr. Helv., 13, 217-231, doi:10.5194/gh-13-217-1958, 1958.

Auerswald, K.: Predicting nutrient enrichment from long-tem average soil loss, Soil Technol., 2, 271-277, 1989.

Baltzer, P.: Besondere Dünge-Vorschriften im Einzugsgebiet des Hallwilersees, Umwelt Aargau, 58, 9-12, 2012.

Barbosa, F. T., Bertol, I., Luciano, R. V., und Gonzalez, A. P.: Phosphorus losses in water and sediments in runoff of the water erosion in oat and vetch crops seed in contour and downhill, Soil Till. Res., 106, 22-28, 2009.
BUWAL: Phosphor in Böden. Standortbestimmung Schweiz, Schriftenreihe Umwelt Nr. 368, 1-174, 2004.

BLW (Hrsg.): Erosionsrisikokarte, Online verfügbar, http://www. agri-gis.admin.ch/?initialState=ERK\&reset_session\&lang=de (Stand: 07. November 2012), 2010.

Dearnaley, M. P.: Direct Measurements of Settling Velocities in the Owen Tube: A Comparison with Gravimetric Analysis, J. Sea Res., 36, 41-47, 1996.

Eijkelkamp Agrisearch Equipment: Gebrauchsanweisung Nasse-Sieb-Methode, Giesebeek, 1-7, Online verfügbar: http://www.eijkelkamp.com/files/media/Gebruiksaanwijzingen/ DU/m1-0813dnasse-sieb-method.pdf (Stand: 29. January 2013), 2008.

Gallet, A., Flisch, R., Ryser, J. P., Frossard, E., and Sinaj, S.: Effect of phosphate fertilization on crop yield and soil phosphorus status, J. Plant Nutr. Soil Sc., 166, 568-578, 2003.

Hübner, R.: Proposed retrofit for sediment settling velocity tubes (SVTs), Geochem. J., 45, 417-419, 2011.

Kemper, W. D. und Rosenau, R. C.: Aggregate Stability and Size Distribution, Herausgeber: Klute, A.: Methods of Soil Analysis. Part 1 - Physical and Mineralogical Methods, Madison, 425-442, 1986.

Prasuhn, V.: Phosphorabschwemmung von Graslandflächen in der Schweiz - Eintragspfade und Massnahmen zur Verminderung, in: 2. Umweltökologisches Symposium, 73-78, 2010.

Prasuhn, V. und Braun, M.: Abschätzung der Phosphor- und Stickstoffverluste aus diffusen Quellen in die Gewässer des Kantons Bern, Schriftenreihe der FAC Liebefeld, Nr. 17., Liebefeld-Bern, 1-113, 1994.

Saunders, W. M. H. und Williams, E. G.: Observations on the determination of total organic phosphorus in soils, J. Soil Sci., 6, 254-267, 1955.

Sharpley, A. N., Menzel, R. G., Smith, S. J., Rhoades, E. D., und Olness, A. E.: The Sorption of Soluble Phosphorus by Soil Material during Transport in Runoff from Cropped and Grassed Watersheds, J. Environ. Qual., 10, 211-215, 1981.

Starr, G. C., Lal, R., Malone, R., Hothem, D., Owens, L., und Kimble, J.: Modeling soil carbon transported by water erosion processes, Land Degrad. Dev., 11, 83-91, 2000.

Stöckli, A.: Dem Hallwilersee geht es immer besser!, Umwelt Aargau, 49, 13-20, 2010. 